\title{
Equal Pay for Unequal Work: Limiting Sabotage in Teams
}

by

Arup Bose, ${ }^{*}$ Debashis Pal, ${ }^{* *}$ and David E. M. Sappington***

\begin{abstract}
We demonstrate the value of "equal pay" policies in teams, even when team members have distinct abilities and make different contributions to team performance. A commitment to compensate all team members in identical fashion eliminates the incentive that each team member otherwise has to sabotage the activities of teammates in order to induce the team owner to implement a more favorable reward structure. The reduced sabotage benefits the team owner, and can secure Pareto gains under plausible circumstances.
\end{abstract}

December 2008

* Indian Statistical Institute (abose@isical.ac.in).

** Department of Economics, University of Cincinnati (pald@email.uc.edu).

*** Department of Economics, University of Florida (sapping@ufl.edu).

We are grateful to the coeditor, three anonymous referees, Noriaki Matsushima, Delphine Prady, and Nicolas Williams for very helpful comments. The first two authors thank the Taft Research Center at the University of Cincinnati for generous research support. 


\section{Introduction.}

It is well known that participants in a tournament can face strong incentives to sabotage the activities of fellow participants (e.g., Dye, 1984; Lazear, 1989; Chen, 2003; Münster, 2007). By sabotaging a competitor's activities, a participant can improve his relative performance and thereby increase the probability that he wins the tournament and collects the associated prize.

It is also well known that very different incentives often prevail in team settings, where only the aggregate performance of the team - not the performance of individual team members - is observable. Consider, for example, the simple setting in which the team project either succeeds or fails, each team member (i.e., each "agent") is risk neutral, no agent has any initial wealth, and the probability that the team project succeeds is a strictly increasing function of the contribution of each agent. In this setting, each agent will optimally receive a positive payment only when the project succeeds. Therefore, given the prevailing wage structure, each agent's expected profit increases as the aggregate probability of success increases. In such a setting, each agent will wish to assist, not sabotage, the operations of his teammates.

Although members of a team often will refrain from sabotage, they will not always do so. We show that an agent can gain from sabotaging the operations of his teammates when the sabotage occurs before the team's owner ("the principal") finalizes the relevant compensation structure. To understand the potential gain from sabotage, suppose one team member agent $\mathrm{A}$ - sabotages the activities of his teammates and thereby increases their operating costs. The sabotage reduces agent A's relative cost of enhancing the aggregate probability of project success. In response to agent A's lower relative cost, the principal optimally secures a larger contribution to project success from agent A. The principal does so by differentially increasing the payment to agent A when the project succeeds. The prospect of securing this increased payment can lead agent A to sabotage the operations of his teammates.

To illustrate how sabotage might arise in practice, consider a setting where a pharma- 
ceutical company charges a team of scientists with the task of discovering a profitable new drug. The likelihood that the team eventually discovers the new drug increases as each scientist on the team increases his non-contractible research effort and/or acquires valuable complementary resources. These resources might include research assistance and/or full knowledge of the interim discoveries of all scientists on the team. During the long-term research process, the pharmaceutical company acquires updated information about conditions in the labor market for research scientists and about the relative skills of the scientists on the team. As it acquires this information, the company adjusts the compensation of the scientists accordingly, in part to ensure that the highly-valued researchers are retained.

In such a setting, one scientist ( $\mathrm{A}$ ") may be tempted to sabotage the operation of another scientist ("B") in order to enhance his own perceived relative talents and thereby secure more favorable compensation. Scientist A's sabotage might take the form of withholding valuable research findings from scientist $B$, for example. Alternatively, scientist A might fail to help recruit a research assistant whose skills and talents are particularly complementary with those of scientist B.

We demonstrate that sabotage often will arise in settings like this one and more generally. In particular, sabotage arises in our model whenever the agents choose their preferred levels of mutual assistance or sabotage before the principal specifies the final terms of the reward structure in the team. In practice, the prospect of renegotiation often limits a principal's ability to commit to the final details of a reward structure before all relevant interaction among team members has been completed. However, we show that the principal can preclude sabotage if she is simply able to commit herself to implement an "equal pay" policy that promises identical payments to all agents. An equal pay policy does not constrain the principal to specify in advance the exact payment that she will ultimately deliver to each agent when the project succeeds. Consequently, the policy affords the principal some ability to adjust payments as the environment changes (e.g., as conditions in the labor market change or as new information about the relative productivities of team members arrives). However, 
by eliminating her ability to differentially favor an agent as his relative cost declines, the principal eliminates the incentive that agents would otherwise have to sabotage the activities of their teammates.

The principal often increases her own expected payoff by precluding sabotage with an equal pay policy. We show that this is the case, for example, when the potential impact of sabotage is sufficiently pronounced and the innate capabilities of the agents are not too disparate. We also identify plausible conditions under which an equal pay policy generates Pareto gains by increasing the expected profit of both the principal and her agents.

Legal mandates and social norms facilitate commitment to equal pay policies in many countries. Indeed, employers often are obligated to deliver the same compensation for comparable performance to workers with similar education, skills, and experience. ${ }^{1}$ Thus, in practice, employers may be able to make binding long-term commitments to equal pay policies even when corresponding promises about specific wage payments are renegotiable.

Our finding that a commitment to an equal pay policy can increase surplus by limiting sabotage complements related findings in the literature. For example, Bevia and Corchon (2006) examine a setting where (in contrast to our model) the outputs produced by individual agents are contractible, and so the distribution of the total output among the agents can reflect their individual contributions. The authors show that sharing rules that are less sensitive to the agents' individual contributions can reduce the time that each agent devotes to sabotaging the operations of other agents. ${ }^{2}$

Other authors observe that equal pay policies can be particularly advantageous in settings where agents experience disutility (or "envy") whenever they receive a lower payoff than

\footnotetext{
${ }^{1}$ See, for example, Borland (1999), Heide (1999), Maatta (2000), and Baker (2004).

${ }^{2}$ Auriol et al. (2002) consider a two-period moral hazard setting in which the abilities of symmetric, riskaverse agents are initially unknown. Favorable first-period performance supports the inference that the agents have high ability, which leads the principal to prefer a more high-powered second-period reward structure. The prospect of facing increased risk in the second period reduces the agents' incentives to deliver superior first-period performance, and so they provide less assistance to one another. To limit this reduced assistance (which is a form of sabotage), the principal optimally commits in advance to implement a relatively low-powered second-period reward structure even when first-period performance is pronounced.
} 
other agents. ${ }^{3}$ Mui (1995) finds that envious agents may sabotage the operation of more successful counterparts if those counterparts are highly and differentially rewarded for their superior individual performance. More equal sharing of the realized surplus serves to limit such sabotage in Mui's model. ${ }^{4}$ Bartling and von Siemens (2007) identify conditions under which an equal sharing rule constitutes the optimal compensation structure to motivate non-contractible effort from envious agents. ${ }^{5}$

Our analysis proceeds as follows. Section 2 reviews the key elements of our model. Section 3 presents our main findings. Section 4 discusses extensions of our basic model. Section 5 concludes and suggests directions for future research. The proofs of all formal conclusions are presented in the Appendix.

\section{The Model.}

We consider a setting where a risk-neutral principal hires two risk-neutral agents - agent A and agent $\mathrm{B}$ - to operate her project. ${ }^{6}$ The project either succeeds or fails. A successful project generates value $V$ for the principal. A failed project generates no value.

Agent $i \in\{A, B\}$ contributes success probability $p_{i}$ to the project. The aggregate probability that the project succeeds $(p)$ is the sum of the success probabilities contributed by the two agents, i.e., $p=p_{A}+p_{B}$. This simple formulation is adopted initially for analytic ease. A formulation that admits interactions among the agents' contributions is considered

\footnotetext{
${ }^{3}$ Empirical research suggests that employers implement limited wage variation in practice in part to avoid perceptions of unfair or inequitable compensation policies. See Blinder and Choi (1990), Agell and Lundberg (1995), Campbell and Kamlani (1997), and Bewley (1999), for example. Farrell and Scotchmer (1988) and Encinosa et al. (2007) report that members of legal and medical partnerships often share profits equally.

${ }^{4}$ Itoh (1991) identifies conditions under which a principal gains by explicitly motivating agents to assist one another. Itoh's work, like the work of Mui (1995) and Bevia and Corchon (2006), differs from ours in part because each agent's individual performance is observable and contractible in these other works. Itoh's primary conclusions, like the conclusions in Auriol et al. (2002), reflect the presumed risk aversion of agents. The agents are risk neutral in our model.

${ }^{5}$ Bartling and von Siemens (2007) abstract from the possibility of sabotage. An equal sharing rule is not necessarily the optimal rule among all possible sharing rules in our model. However, the principal in our model often prefers to commit to such a rule in order to limit sabotage rather than retain the flexibility to fully tailor the reward structure to the agents' distinct capabilities.

${ }^{6}$ Lemma 1 in section 3 identifies conditions under which the principal prefers to hire two agents than to hire a single agent.
} 
in section 4.

An agent will work for the principal as long as he anticipates non-negative profit from doing so. An agent's profit is the difference between the payment he receives from the principal and the cost he incurs working for the principal. Agent $i$ incurs cost $\frac{k_{i}}{\theta}\left(p_{i}\right)^{\theta}$ when he delivers success probability $p_{i}$, where $k_{i} \in\left[\underline{k}_{i}, \bar{k}_{i}\right], 0<\underline{k}_{i}<\bar{k}_{i}$, and $\theta>2 .^{7}$ Therefore, each agent's cost is an increasing, convex function of the success probability that he contributes.

We will analyze the incentive of each agent to assist or to hinder the operations of his teammate. We assume that agent $i$ can, at no personal cost, choose his preferred level of $k_{j} \in\left[\underline{k}_{j}, \bar{k}_{j}\right]$ for $j \neq i, i, j \in\{A, B\}$. Thus, one might view $k_{j}^{o} \in\left[\underline{k}_{j}, \bar{k}_{j}\right]$ as agent $j$ 's innate operating cost. (For expositional ease, we will often refer to $k_{i}$ as agent $i$ 's "cost" or "operating cost," even though $k_{i}$ is actually a parameter of the agent's cost function.) Agent $i$ can reduce $k_{j}$ below $k_{j}^{o}$ by providing assistance to agent $j$. Alternatively, agent $i$ can increase $k_{j}$ above $k_{j}^{o}$ by sabotaging agent $j$ 's activities. The bounds on feasible assistance $\left(k_{j}^{o}-\underline{k}_{j}\right)$ and sabotage $\left(\bar{k}_{j}-k_{j}^{o}\right)$ might reflect, for example, technological considerations or the limited amount of time that agent $i$ has available to assist or to sabotage his teammate's operations. We assume that the agents determine simultaneously and independently the level of assistance (or sabotage) that they will deliver.

Neither the individual contributions $\left(p_{i}\right)$ of the team members nor the final operating $\operatorname{costs}\left(k_{i} \in\left[\underline{k}_{i}, \bar{k}_{i}\right]\right)$ of the agents are contractible. ${ }^{8}$ Therefore, even though she ultimately observes each agent's operating cost, the principal cannot base payments directly on the level of these costs. Payments can only reflect the ultimate success or failure of the project. $T_{i}$ will denote the payment the principal delivers to agent $i$ when the project succeeds. The

\footnotetext{
${ }^{7}$ This class of cost functions admits closed form solutions for all relevant variables, and thereby facilitates direct comparisons of distinct institutional regimes. While not completely general, this cost structure admits a wide variety of plausible cost functions.

${ }^{8}$ Final operating costs $k_{j} \in\left[\underline{k}_{j}, \bar{k}_{j}\right]$ (and thus the level of assistance that each agent provides) may not be contractible, for instance, because of the detailed, idiosyncratic knowledge of the working environment that is required to distinguish among the many factors that affect operating costs. However, sabotage in excess of $\bar{k}_{j}-k_{j}^{o}$ may be so egregious that it is readily observed and documented, and thus contractible.
} 
principal makes the minimum possible payment (0) to each agent when the project fails. ${ }^{9}$ This minimum payment might reflect the agent's limited wealth or legal limits on the agent's liability, for example (e.g., Sappington, 1983). The principal seeks to maximize the expected difference between the value she derives from the project and the payments she makes to the agents.

Given the prevailing configuration of the agents' costs $\left(k_{A}, k_{B}\right)$, the principal's problem, $[\mathrm{P}]$, is the following:

$$
\underset{\left\{T_{A}, T_{B}\right\}}{\operatorname{Maximize}}\left(p_{A}+p_{B}\right)\left(V-T_{A}-T_{B}\right)
$$

subject to:

$$
\begin{aligned}
& \left(p_{A}+p_{B}\right) T_{i}-\frac{k_{i}}{\theta}\left(p_{i}\right)^{\theta} \geq 0 \text { for } i=A, B ; \\
& p_{A}=\underset{p}{\arg \max }\left\{\left(p+p_{B}\right) T_{A}-\frac{k_{A}}{\theta}(p)^{\theta}\right\} ; \\
& p_{B}=\underset{p}{\arg \max }\left\{\left(p_{A}+p\right) T_{B}-\frac{k_{B}}{\theta}(p)^{\theta}\right\} ; \text { and } \\
& 0 \leq p_{A}+p_{B} \leq 1 .
\end{aligned}
$$

Expression (1) reflects the principal's desire to maximize the expected difference between the value she derives from the project and the payments she makes to the agents. Inequality (2) ensures that the agents receive non-negative (expected) profit, in equilibrium. ${ }^{10}$ Equations (3) and (4) identify the equilibrium success probabilities that agents A and B, respectively, contribute to the project (simultaneously and independently). Inequality (5) ensures that the aggregate probability of success is well defined.

\section{Findings.}

As noted in the Introduction, agents often have strong incentives to sabotage each other's activities when they compete in tournaments. By diminishing a competitor's performance,

\footnotetext{
${ }^{9} \mathrm{It}$ is readily shown that it is optimal for the principal to deliver the smallest possible payment to each agent when the project fails. This payment structure minimizes the agents' rent for any given level of incentive they face to increase the probability of project success.

${ }^{10}$ For expositional ease, the term "profit" often will be employed in place of "expected profit."
} 
an agent can improve his own relative performance, and thereby increase the probability that he wins the tournament.

Very different incentives can arise when agents operate in teams. In team settings like the one considered here, the principal cannot reward agents according to their relative performance because individual contributions to aggregate performance are not contractible. Payments can only reflect the observed success or failure of the entire team project. To motivate both agents to contribute to the success of the team project, the principal optimally delivers a strictly positive payment to each agent whenever the project succeeds. Therefore, given the prevailing reward structure, each agent's expected net payoff increases as the aggregate probability of success increases. Consequently, if the principal is able to make a binding commitment to the reward structure that will ultimately prevail before the agents decide how much assistance to deliver, ${ }^{11}$ each agent will maximize his own profit by assisting his teammate to the greatest extent possible. The assistance reduces the teammate's operating cost, which leads him to deliver a higher success probability. This conclusion is recorded formally as Proposition 1.

Proposition 1. In the benchmark setting where the principal can make a binding commitment to the final terms of the reward structure before each agent chooses the level of assistance that he delivers to his teammate, each agent will provide the maximum feasible assistance to his teammate, i.e., agent $A$ will set $k_{B}=\underline{k}_{B}$ and agent $B$ will set $k_{A}=\underline{k}_{A} \cdot{ }^{12}$

There are some settings where, as presumed in Proposition 1, a principal may be able to make a binding commitment to the details of a reward structure before each agent decides how much assistance (or sabotage) he will deliver. This timing might arise, for example, in a short-term employment setting where the employer first specifies the wage structure and

\footnotetext{
${ }^{11}$ For expositional ease, the ensuing discussion often will refer to an agent's decision about how much assistance or sabotage to deliver simply as the agent's decision about how much assistance to deliver.

${ }^{12}$ Following the techniques employed in the proof of Lemma 1, it is readily shown that the principal prefers to hire two agents rather than just one agent in this benchmark setting (given the maintained assumption that $\theta>2$ ).
} 
each individual employee then decides how much of his scarce time to devote to physically assisting a fellow employee or to distracting the employee from his assigned task.

Alternative timings are plausible in different settings, though. Consider, for example, a long-term employment relationship in which labor market conditions and employee productivities change over time, and where employers can adjust wages to attract or retain valued workers and to induce more efficient configurations of employee effort. ${ }^{13}$ Suppose further that each employee can mentor his fellow workers and help them to improve their operating skills. Alternatively, the employee can make a concerted effort to reduce the long-term productivity of fellow workers, perhaps by blocking their efforts to acquire valuable skills or to secure complementary resources (as in the pharmaceutical example discussed in the Introduction).

In settings like these, it is reasonable to view the agents as choosing their preferred levels of assistance before the principal learns the agents' exact capabilities and specifies the final details of the team's reward structure. When this timing prevails, rational agents will consider the impact of their actions on the reward structure that ultimately will be implemented. The ensuing analysis will focus on this setting.

To avoid cases of limited interest, we will restrict attention to settings where the principal prefers to hire two agents rather than just one agent. ${ }^{14}$ Conceivably, the principal might prefer to hire a single agent, and thereby avoid both sabotage and free-rider problems. A free-rider problem can arise when an agent reduces his contribution to the aggregate success probability, knowing that the principal cannot distinguish between his contribution and the contribution of his teammate. On the other hand, the principal might prefer to hire two agents because it is more costly for a single agent to secure a given aggregate success probability than it is for two agents to secure the same success probability. The increased

\footnotetext{
${ }^{13}$ In principle, an employer might propose a long-term employment contract in which wages do not change as labor market conditions and employee productivities change. In practice, though, such contracts are likely to be renegotiated when Pareto-improving opportunites arise.

${ }^{14}$ For analytic ease, we assume that the team consists of at most two agents. The possibility of expanded team membership is discussed in section 5 .
} 
cost when the team consists of a single agent reflects the convex cost structure $(\theta>2)$ under which each agent operates.

Lemma 1 reports that the principal prefers to hire two agents rather than a single agent whenever the diminishing returns that each agent faces in enhancing the probability of project success are sufficiently pronounced (i.e., whenever $\theta$ is sufficiently large) relative to the maximum feasible level of sabotage. The lemma refers to $\underline{k} \equiv \min \left\{\underline{k}_{A}, \underline{k}_{B}\right\}$ and $\bar{k}$ $\equiv \max \left\{\bar{k}_{A}, \bar{k}_{B}\right\}$. The inequality in Lemma 1 is assumed to hold throughout the ensuing analysis.

Lemma 1. The principal prefers to hire two agents rather than a single agent if $\theta>2+$ $\frac{\ln (\bar{k} / \underline{k})}{\ln 2}$.

Lemma 2 now characterizes the reward structure that the principal will implement when she does not specify the terms of the final compensation schedule until after the agents have chosen their preferred levels of assistance or sabotage. The lemma also reports the success probability $\left(p_{i}\right)$ that each agent will deliver under this optimal reward structure, given their prevailing costs $\left(k_{i}\right)$. The prevailing costs reflect the equilibrium levels of assistance and sabotage.

Lemma 2. $T_{i}=V \theta^{-1}\left(1+\left(\frac{k_{i}}{k_{j}}\right)^{\frac{1}{\theta-2}}\right)^{-1}$ and $p_{i}=V^{\frac{1}{\theta-1}}\left(\theta k_{i}\left(1+\left(\frac{k_{i}}{k_{j}}\right)^{\frac{1}{\theta-2}}\right)\right)^{-\frac{1}{\theta-1}}$ at the solution to $[P]$, for $i, j \in\{A, B\}, i \neq j$.

Lemma 2 reveals that the principal optimally provides a larger reward for success $\left(T_{i}\right)$ to agent $i$ as the cost $\left(k_{j}\right)$ of agent $j$ increases. Furthermore, the rate at which $T_{i}$ increases as $k_{j}$ increases exceeds the corresponding rate at which the success probability that agent $j$ ultimately contributes $\left(p_{j}\right)$ declines. Thus, on balance, each agent gains as he hinders the activities of his teammate because of the resulting impact on the equilibrium reward structure, as Proposition 2 reports. 
Proposition 2. Suppose each agent chooses the level of assistance that he delivers to his teammate before the principal makes any commitment regarding the terms of the reward structure. Then each agent will sabotage his teammate's activities to the greatest extent possible, i.e., agent $A$ will set $k_{B}=\bar{k}_{B}$ and agent $B$ will set $k_{A}=\bar{k}_{A}$.

Proposition 2 reflects the fact that the principal effectively has two sources from which she can procure an essential input (i.e., success probability) - agent A and agent B. As agent B's operating cost $\left(k_{B}\right)$ increases, agent A becomes a relatively less costly source of the essential input. Consequently, the principal optimally secures more of the input from the less costly source by increasing the payment that she makes to agent A when the project succeeds. The prospect of securing an increased reward for success leads each agent to sabotage his teammate's activities to the maximum extent possible when the agents choose their preferred levels of assistance before the principal commits to the terms of the reward structure.

Propositions 1 and 2 reveal that the principal often would gain if she could credibly commit to an immutable, long-term reward structure before the agents chose their preferred levels of assistance. In practice, though, long-term binding commitments to all relevant details of a reward structure can be difficult to ensure. Ongoing changes in labor market conditions and worker productivities can compel employers to modify compensation structures in order to retain valued employees and to allocate aggregate effort supplies more efficiently, for example. A question that arises, then, is whether the principal can avoid the losses that sabotage imposes even when she cannot make a credible long-term commitment to all relevant details of a reward structure.

Proposition 3 reports that the principal can avoid these losses if she can commit to implement an egalitarian reward structure. An egalitarian reward structure in our model is one that always compensates the two agents in identical fashion. ${ }^{15} \mathrm{~A}$ commitment to an egali-

${ }^{15}$ Thus, $T_{A}=T_{B}$ under an egalitarian reward structure. Using techniques analogous to those employed in the proof of Lemma 1 , it is readily shown that when she implements an egalitarian reward structure, the principal prefers to hire two agents rather than one agent if $\theta>2+\frac{\ln \left(\underline{k}_{x} / \underline{k}_{n}\right)}{\ln 2}$, where $\underline{k}_{x} \equiv \max \left\{\underline{k}_{A}, \underline{k}_{B}\right\}$ and $\underline{k}_{n} \equiv \min \left\{\underline{k}_{A}, \underline{k}_{B}\right\}$. 
tarian reward structure does not eliminate the principal's ability to alter the compensation schedule as environmental conditions change. The commitment only obligates the principal to treat the two agents symmetrically. Thus, after committing to implement an egalitarian reward structure, the principal can wait until after the agents have implemented their preferred levels of assistance before specifying the (identical) payment for success that she will deliver to the two agents. As noted in the Introduction, a commitment to implement an egalitarian reward structure often is facilitated in practice by legal obligations to deliver the same compensation for comparable performance to workers with similar observable credentials.

Proposition 3. Suppose the principal commits to implement an egalitarian reward structure before each agent chooses the level of assistance that he delivers to his teammate. Then each agent will provide the maximum feasible assistance to his teammate, i.e., agent $A$ will set $k_{B}=\underline{k}_{B}$ and agent $B$ will set $k_{A}=\underline{k}_{A}$.

A commitment to an egalitarian reward structure alters the incentive of an agent (agent A, for example) to sabotage his teammate's operation. Agent A can reduce his relative cost by increasing agent B's cost $\left(k_{B}\right)$. However, when she is committed to an egalitarian reward structure, the principal cannot respond to the reduction in agent A's relative cost by differentially increasing agent A's payment for success, as she would in the absence of the commitment. Consequently, the primary effect of increasing $k_{B}$ in the presence of an egalitarian reward structure is to reduce agent B's contribution, which reduces the aggregate equilibrium probability of success $(p)$. Because the reduction in $p$ reduces agent A's expected profit, the agent will not sabotage his teammate's activities. Instead, agent A will deliver the maximum possible assistance to agent B in order to increase his own profit by increasing the aggregate probability of success.

The increased assistance induced by a commitment to an egalitarian reward structure benefits the principal by increasing the probability that the project succeeds. This benefit 
often outweighs any loss that the principal incurs from an inability to tailor payments to the distinct capabilities of the agents. As Proposition 4 reports, the benefit exceeds the loss whenever there is any overlap in the range of possible costs for the two agents.

Proposition 4. Suppose $\left(\underline{k}_{A}, \bar{k}_{A}\right) \cap\left(\underline{k}_{B}, \bar{k}_{B}\right) \neq \varnothing$ and suppose each agent chooses his preferred level of assistance before the principal specifies the precise rewards for success. Then the principal's profit is strictly higher when she commits herself ex ante to implement an egalitarian reward structure than when she makes no such commitment.

To understand the principal's preference for an egalitarian reward structure under the condition specified in Proposition 4 , let $\widetilde{k}$ denote a $k$ in $\left(\underline{k}_{A}, \bar{k}_{A}\right) \cap\left(\underline{k}_{B}, \bar{k}_{B}\right) .{ }^{16}$ The principal can ensure that both agents operate with a cost that is below $\widetilde{k}$ by committing to implement an egalitarian reward structure. As Proposition 3 indicates, this commitment induces each agent to deliver the maximum possible assistance to his teammate. Consequently, agent $i \in\{A, B\}$ will operate with cost $\underline{k}_{i}<\widetilde{k}$. In contrast, if the principal does not commit herself to an egalitarian reward structure, each agent will increase his teammate's cost above $\widetilde{k}$ in an attempt to secure differentially favorable treatment from the principal. The resulting high costs will reduce the principal's profit below the level that she would secure if both agents operated with cost $\widetilde{k}$. This profit is less than the profit the principal secures when agent A operates with cost $\underline{k}_{A}<\widetilde{k}$ and when agent $\mathrm{B}$ operates with $\operatorname{cost} \underline{k}_{B}<\widetilde{k}$. The principal can ensure this higher level of profit by committing to an egalitarian reward structure. ${ }^{17}$

The principal's preference for an egalitarian reward structure identified in Proposition 4 is strong in at least two respects. First, the preference holds whenever there is any overlap in the agents' cost structures, however slight that overlap might be. ${ }^{18}$ Second, the preference

${ }^{16}$ Because $\widetilde{k}$ is contained in the (non-empty) intersection of $\left(\underline{k}_{A}, \bar{k}_{A}\right)$ and $\left(\underline{k}_{B}, \bar{k}_{B}\right), \widetilde{k}$ strictly exceeds the minimum possible cost of both agents, i.e., $\widetilde{k}>\max \left\{\underline{k}_{A}, \underline{k}_{B}\right\}$.

${ }^{17}$ Although the principal often benefits from committing to implement an egalitarian reward structure, she would benefit even more if she were able to commit to the details of the final reward structure after she learns the agents' innate costs but before the agents choose their preferred levels of assistance. Such complete commitment would enable the principal to both avoid sabotage (recall Proposition 1) and tailor the prevailing reward structure to the individual capabilities of the two agents (as indicated in Lemma 2).

${ }^{18}$ While the overlap identified in Proposition 4 is sufficient to ensure that the principal prefers to commit 
holds regardless of the magnitudes of the maximum feasible levels of assistance and sabotage.

Tables 1 and 2 and Figures 1 and 2 help to illustrate the magnitude of the gains the principal can secure by committing to an egalitarian reward structure. The tables consider nine combinations of equilibrium $\operatorname{costs}\left(k_{A}, k_{B}\right)$ for the agents in a setting where the value of success $(V)$ is 4 and the agents' cost parameter $\theta$ is also 4 . Table 1 reports the equilibrium contributions of the two agents $\left(p_{A}\right.$ and $\left.p_{B}\right)$, the aggregate probability of project success $(p)$, the payments to the two agents when the project succeeds $\left(T_{A}\right.$ and $\left.T_{B}\right)$, and the profits of agent $\mathrm{A}\left(R_{A}\right)$, agent $\mathrm{B}\left(R_{B}\right)$, and the principal $(\pi)$ in the absence of any commitment to an egalitarian reward structure. Table 2 provides the corresponding measures when the principal commits to implement an egalitarian reward structure. ${ }^{19}$

Tables 1 and 2 reveal that if $\underline{k}_{A}=\underline{k}_{B}=8$ and $\bar{k}_{A}=\bar{k}_{B}=9$, the principal's profit increases by approximately $4 \%$ when she commits herself to implement an egalitarian reward structure. This reward structure induces the agents to deliver the maximum level of assistance to each other (recall Proposition 3), and so both agents operate with cost parameter $k=8$. As reported in the first cell in the first row of Table 2, the principal's expected profit in this case is 2.381. Absent a commitment to an egalitarian reward structure, the agents undertake the maximum level of sabotage (recall Proposition 2), and so both agents operate with cost parameter $k=9$. As reported in the middle cell in the second row of Table 1, the principal's expected profit in this case is 2.289. Therefore, the principal's expected profit increases by $4 \%\left(\approx 100\left[\frac{2.381-2.289}{2.289}\right] \%\right)$ when she commits herself to implement an egalitarian reward structure in this setting. The corresponding increase is almost $8 \%(\approx$ $\left.100\left[\frac{2.381-2.210}{2.210}\right] \%\right)$ when $\underline{k}_{A}=\underline{k}_{B}=8$ and $\bar{k}_{A}=\bar{k}_{B}=10$. The larger increase in profit in this latter case reflects the more pronounced reduction in sabotage that the commitment to an egalitarian reward structure ensures.

to implement an egalitarian reward structure, overlap is not necessary for this preference to arise. This conclusion is illustrated in Table 3 (below).

${ }^{19}$ Equilibrium profit levels under the optimal egalitarian reward structure are denoted by the subscript "e" in Table 2 (and in Table 3). 
Figure 1 illustrates the corresponding gains for the principal as the maximum possible cost for the two agents $\left(\bar{k}_{A}=\bar{k}_{B}\right)$ varies between 8 and 16. The figure reveals that the principal's gain from committing to implement an egalitarian reward structure increases as the maximum level of sabotage $\left(\bar{k}_{A}-\underline{k}_{A}=\bar{k}_{B}-\underline{k}_{B}\right)$ increases. ${ }^{20}$ The commitment increases the principal's expected profit by approximately 26 percent when sabotage can double the agents' cost (from $\underline{k}_{A}=\underline{k}_{B}=8$ to $\bar{k}_{A}=\bar{k}_{B}=16$ ) in this setting.

Figure 2 illustrates how the principal's expected gain from committing to implement an egalitarian reward structure changes as the agents' cost parameter $\theta$ changes in the setting where $V=4, \underline{k}_{A}=\underline{k}_{B}=8$, and $\bar{k}_{A}=\bar{k}_{B}=10$. The figure indicates that the principal's gain declines as $\theta$ increases in this setting (as it does more generally). The reduced gain reflects the fact that each agent's cost of delivering a specified contribution to project success, $\frac{k_{i}}{\theta}\left(p_{i}\right)^{\theta}$, declines as $\theta$ increases, holding $k_{i}$ constant. ${ }^{21}$ Therefore, the reduction in $k_{i}$ achieved by committing to implement an egalitarian reward structure secures a smaller reduction in the agents' operating costs, ${ }^{22}$ and so is less advantageous for the principal. ${ }^{23}$

The agents, like the principal, can gain when the principal commits to implement an egalitarian reward structure. To illustrate this conclusion, consider the setting where $V=$ $\theta=4, k_{A} \in[8,9]$, and $k_{B} \in[9,10]$. Tables 1 and 2 reveal that the commitment to an egalitarian reward structure in this setting increases agent A's profit by 1.2\% (from .336 to .340 ) and agent B's profit by $6.5 \%$ (from .321 to .342 ) as it increases the principal's profit. ${ }^{24}$

${ }^{20}$ The solid dots in Figure 1 and in subsequent figures represent explicit numerical solutions. The lines in the figures connect these dots.

${ }^{21} \frac{\partial}{\partial \theta}\left\{\frac{k_{i}}{\theta}\left(p_{i}\right)^{\theta}\right\}=k_{i}\left(\frac{1}{\theta}\left(p_{i}\right)^{\theta} \ln p_{i}-\frac{1}{\theta^{2}}\left(p_{i}\right)^{\theta}\right)=\frac{k_{i}\left(p_{i}\right)^{\theta}}{\theta^{2}}\left(\theta \ln p_{i}-1\right)<0$ for all $p_{i} \in(0,1]$.

$22 \frac{\partial}{\partial k_{i}}\left\{\frac{\partial}{\partial \theta}\left(\frac{k_{i}}{\theta}\left(p_{i}\right)^{\theta}\right)\right\}=\frac{\left(p_{i}\right)^{\theta}}{\theta^{2}}\left(\theta \ln p_{i}-1\right)<0$ for all $p_{i} \in(0,1]$.

${ }^{23}$ Equations (29), (46), and (48) in the Appendix reveal that the principal's expected profit is proportional to $V^{\frac{\theta}{\theta-1}}$ both when she commits herself to implement an egalitarian reward structure and when she does not make this commitment. Therefore, the percentage increase in the principal's expected profit from committing to an egalitarian reward structure does not vary with $V$ in this setting. This percentage increase is approximately $14.5 \%$ when $\underline{k}_{A}=\underline{k}_{B}=8, \bar{k}_{A}=\bar{k}_{B}=10$, and $\theta=4$.

${ }^{24}$ When the principal commits to implement an egalitarian reward structure in this setting, each agent provides the maximum level of assistance to his counterpart. Therefore, agent A operates with cost parameter $k_{A}=8$ and agent $\mathrm{B}$ operates with cost parameter $k_{B}=9$. The middle cell in the top row of Table 2 indicates that agent A's expected profit is .340 and agent B's expected profit is .342 in this case. Absent such a commitment, sabotage drives agent A's cost parameter to $k_{A}=9$ and agent B's cost 
More generally, the commitment to an egalitarian reward structure always secures Pareto gains if the set of feasible costs is the same for the two agents, as Proposition 5 reports.

Proposition 5. Suppose $\underline{k}_{A}=\underline{k}_{B}$ and $\bar{k}_{A}=\bar{k}_{B}$. Then the profit of the principal and the profit of both agents are strictly higher when the principal commits herself ex ante to implement an egalitarian reward structure than when she makes no such commitment.

When the set of feasible costs is the same for the two agents, they both operate with cost $\underline{k}_{A}\left(=\underline{k}_{B}\right)$ when the principal commits to an egalitarian payment structure. In contrast, they both operate with cost $\bar{k}_{A}\left(=\bar{k}_{B}\right)$ when the principal makes no such commitment. It is readily shown that when they have the same final operating cost (i.e., when $k_{A}=k_{B}$ ), the profit that each agent secures in equilibrium increases as his operating cost declines. Therefore, both agents and the principal benefit from the lower symmetric costs that arise when the two agents have the same set of feasible costs and when the principal ensures the lowest feasible cost by committing herself to implement an egalitarian reward structure.

Although a commitment to an egalitarian reward structure can secure Pareto gains under the conditions cited in Proposition 5 and more generally, such gains do not always arise. A particularly productive agent may be harmed by a commitment to an egalitarian reward structure. The agent benefits from the increased assistance that an egalitarian reward structure engenders. However, he may be harmed when the principal reduces the payment for success that she otherwise would deliver to the more productive agent. The principal optimally reduces this payment when she is committed to deliver the same payment to both agents.

Table 3 illustrates this more general conclusion. The table reports the equilibrium outcomes when $V=\theta=4$ and when the cost of the more productive agent (agent A) can vary between 8.0 and 8.2 while the cost of the less productive agent (agent B) can vary between 9.8 and 10.0. In the absence of a commitment to an egalitarian reward structure, expected payoff is .366 and agent B's expected profit is .321 in this case. 
the principal optimally pays agent A more than she pays agent B when the project succeeds $\left(T_{A}=.525>.475=T_{B}\right)$. Doing so induces the more productive agent to deliver a relatively large contribution $\left(p_{A}=.4\right)$ to the aggregate probability of success. When a commitment to an egalitarian reward structure compels the principal to deliver the same payments to the two agents, she optimally reduces the payment to agent $\mathrm{A}$ (from .525 to .50). This payment reduction reduces agent A's profit (from .348 to .334), despite the cost reduction (from 8.2 to 8.0) that he secures under an egalitarian reward structure.

\section{Non-Independent Contributions.}

The analysis to this point has focused on settings in which the contributions of the two agents are independent. Before concluding, we briefly consider the extent to which our key qualitative conclusions persist in more general settings.

To do so, suppose the team project succeeds with probability $p=p_{A}+p_{B}+\gamma p_{A} p_{B}$ when agent $\mathrm{A}$ contributes success probability $p_{A}$ and agent $\mathrm{B}$ contributes success probability $p_{B} . \quad \gamma$ is a parameter that captures the interactions between the agents' contributions. When $\gamma$ is positive, the contributions of the two agents are complements. In other words, an increased contribution by one agent increases the rate at which the aggregate probability of success increases as the contribution of the other agent increases. When $\gamma$ is negative, the contributions of the two agents are substitutes in the sense that an increased contribution by one agent reduces the rate at which the aggregate probability of success increases as the contribution of the other agent increases.

Sabotage can be more attractive to an agent when the agents' contributions are substitutes $(\gamma<0)$ than when they are independent $(\gamma=0)$. The increased attraction arises because, in addition to garnering more favorable treatment from the principal (as explained above), sabotage of a teammate reduces his equilibrium contribution to the aggregate probability of project success, and thereby increases the rate at which the agent's own contribution increases the aggregate success probability when $\gamma<0 .{ }^{25}$

$\overline{{ }^{25} \text { Formally, } \frac{\partial p}{\partial p_{i}}=1+\gamma p_{j} \text { increases as } p_{j}}$ decreases when $\gamma<0$. 
In contrast, sabotage can be less attractive to agents when their contributions are complements (i.e., when $\gamma>0$ ). Sabotage of a teammate's operations reduces the teammate's equilibrium contribution to the aggregate probability of project success $(p)$. When $\gamma>0$, this reduced contribution reduces the rate at which the agent's own contribution increases $p$, which can reduce the agent's expected profit. ${ }^{26}$ Consequently, the agents may deliver the maximum feasible level of assistance even if the principal does not commit herself to implement an egalitarian reward structure when $\gamma$ is sufficiently large. Under such circumstances, the principal will not make this commitment because doing so would only limit her ability to induce a more efficient configuration of agent contributions without fostering increased assistance among team members.

Figure 3 illustrates this conclusion in a setting where $V=\theta=4, \underline{k}_{A}=8, \bar{k}_{A}=8.5$, $\underline{k}_{B}=12$, and $\bar{k}_{B}=12.5$. The figure presents the percentage increase in the principal's expected profit from committing to implement an egalitarian reward structure $\left(100\left[\frac{\pi^{e}-\pi}{\pi}\right]\right)$ as $\gamma$ varies between -0.5 and +0.5 . Figure 3 reveals that the principal prefers to commit to an egalitarian reward structure (in order to eliminate sabotage) when $\gamma \leq .15$ in this setting. In contrast, the principal prefers no such commitment when $\gamma \geq .16$ because sabotage does not arise in equilibrium even when payments to the agents can differ. ${ }^{27}$

\section{Conclusions.}

We have shown that sabotage can arise in teams, just as it can in tournaments. An agent reduces his relative cost when he sabotages the operation of a teammate. In response, the principal secures a larger contribution from the relatively more capable agent by rewarding him more generously when the project succeeds. Each agent finds it profitable to sabotage the operation of his teammate in order to secure this increased payment from the principal.

The principal can prevent such sabotage by committing herself to an egalitarian reward

${ }^{26}$ Formally, $\frac{\partial p}{\partial p_{i}}=1+\gamma p_{j}$ declines as $p_{j}$ declines when $\gamma>0$.

${ }^{27}$ The data presented in Figure 3 reflect numerical solutions of the necessary conditions for a solution to the principal's problem in the setting where $p=p_{A}+p_{B}+\gamma p_{A} p_{B}$. It is readily verified that the principal's problem is concave in this setting, and so the necessary conditions are also sufficient. 
structure. Furthermore, the principal often gains more from the reduced sabotage than she loses from the inability to tailor the reward structure to the individual capabilities of team members. This is the case, for example, whenever there is any overlap of the feasible operating costs of the two agents. The agents also can gain from the reduced sabotage that an egalitarian reward structure secures.

Extensions of our simple model merit investigation. For example, explicit costs of assistance and sabotage might be considered. When it is costly for an agent to sabotage the activities of a teammate, the agent may not implement the maximum feasible level of sabotage. Even in this case, though, an egalitarian reward structure is likely to be valuable in limiting sabotage whenever the personal cost that an agent incurs in implementing sabotage is sufficiently small.

For simplicity, we have only considered the possibility that the principal might commit to an egalitarian reward structure. More generally, suppose the principal can make a credible commitment ex ante to limit to $\Delta$ the difference in the payments that are ultimately delivered to the two agents. The principal may prefer a relatively small, but strictly positive, value of $\Delta$ in this setting when sabotage is costly for the agents to implement. A small but positive value of $\Delta$ can limit sabotage without eliminating the principal's ability to tailor the reward structure to the agents' capabilities. Future research might analyze the principal's preferred value of $\Delta$.

Expanded team membership also warrants consideration. When a team consists of more than two agents, each agent will have to decide how to allocate among his teammates the total assistance or sabotage that he delivers. An agent may find it profitable to assist some teammates and sabotage the operations of other teammates, for example. Settings in which multiple teams operate simultaneously might also be analyzed. It would be interesting to determine whether competition among teams with egalitarian reward structures generates an efficient allocation of agents among teams. It would also be interesting to assess how competition for talented agents affects the incentives of principals to implement egalitarian 
or non-egalitarian reward structures.

In closing, we mention three empirical predictions of our model. First, sabotage will be less prevalent in long-term team settings where (perhaps because of prevailing legal mandates) equal pay policies prevail than in corresponding settings where non-egalitarian reward structures prevail. Second, in settings where equal pay policies do not prevail, sabotage will tend to be more pronounced when the non-contractible contributions of team members are substitutes than when they are complements. Third, in jurisdictions where equal pay policies are not mandated but employers can credibly commit to such policies, the policies are more likely to be implemented when the opportunities for sabotage and the impacts of sabotage are pronounced and when variation in the innate capabilities of workers is limited. Tests of these empirical predictions of our model await future research. 


\begin{tabular}{|c|c|c|c|}
\hline & $k_{B}=8.0$ & $k_{B}=9.0$ & $k_{B}=10.0$ \\
\hline$k_{A}=8.0$ & $\begin{array}{c}p_{A}=0.397 \\
p_{B}=0.397 \\
p=0.794 \\
T_{A}=0.500 \\
T_{B}=0.500 \\
R_{A}=0.347 \\
R_{B}=0.347 \\
\pi=2.381\end{array}$ & $\begin{array}{c}p_{A}=0.401 \\
p_{B}=0.378 \\
p=0.778 \\
T_{A}=0.515 \\
T_{B}=0.485 \\
R_{A}=0.349 \\
R_{B}=0.332 \\
\pi=2.335\end{array}$ & $\begin{aligned} p_{A} & =0.404 \\
p_{B} & =0.361 \\
p & =0.766 \\
T_{A} & =0.528 \\
T_{B} & =0.472 \\
R_{A} & =0.351 \\
R_{B} & =0.319 \\
\pi & =2.297\end{aligned}$ \\
\hline$k_{A}=9.0$ & $\begin{array}{c}p_{A}=0.378 \\
p_{B}=0.401 \\
p=0.778 \\
T_{A}=0.485 \\
T_{B}=0.515 \\
R_{A}=0.332 \\
R_{B}=0.349 \\
\pi=2.335\end{array}$ & $\begin{array}{c}p_{A}=0.382 \\
p_{B}=0.382 \\
p=0.763 \\
T_{A}=0.500 \\
T_{B}=0.500 \\
R_{A}=0.334 \\
R_{B}=0.334 \\
\pi=2.289\end{array}$ & $\begin{aligned} p_{A} & =0.385 \\
p_{B} & =0.365 \\
p & =0.750 \\
T_{A} & =0.513 \\
T_{B} & =0.487 \\
R_{A} & =0.336 \\
R_{B} & =0.321 \\
\pi & =2.250\end{aligned}$ \\
\hline$k_{A}=10.0$ & $\begin{array}{c}p_{A}=0.361 \\
p_{B}=0.404 \\
p=0.766 \\
T_{A}=0.472 \\
T_{B}=0.528 \\
R_{A}=0.319 \\
R_{B}=0.351 \\
\pi=2.297\end{array}$ & $\begin{array}{c}p_{A}=0.365 \\
p_{B}=0.385 \\
p=0.750 \\
T_{A}=0.487 \\
T_{B}=0.513 \\
R_{A}=0.321 \\
R_{B}=0.336 \\
\pi=2.250\end{array}$ & $\begin{array}{c}p_{A}=0.368 \\
p_{B}=0.368 \\
p=0.737 \\
T_{A}=0.500 \\
T_{B}=0.500 \\
R_{A}=0.322 \\
R_{B}=0.322 \\
\pi=2.210\end{array}$ \\
\hline
\end{tabular}

Table 1. Outcomes With No Commitment to an Egalitarian Reward Structure when $V=\theta=4$. 


\begin{tabular}{|c|c|c|c|}
\hline & $k_{B}=8.0$ & $k_{B}=9.0$ & $k_{B}=10.0$ \\
\hline$k_{A}=8.0$ & $\begin{aligned} p_{A} & =0.397 \\
p_{B} & =0.397 \\
p & =0.794 \\
T_{A} & =0.500 \\
T_{B} & =0.500 \\
R_{A}^{e} & =0.347 \\
R_{B}^{e} & =0.347 \\
\pi^{e} & =2.381\end{aligned}$ & $\begin{aligned} p_{A} & =0.397 \\
p_{B} & =0.382 \\
p & =0.778 \\
T_{A} & =0.500 \\
T_{B} & =0.500 \\
R_{A}^{e} & =0.340 \\
R_{B}^{e} & =0.342 \\
\pi^{e} & =2.335\end{aligned}$ & $\begin{aligned} p_{A} & =0.397 \\
p_{B} & =0.368 \\
p & =0.765 \\
T_{A} & =0.500 \\
T_{B} & =0.500 \\
R_{A}^{e} & =0.333 \\
R_{B}^{e} & =0.337 \\
\pi^{e} & =2.296\end{aligned}$ \\
\hline$k_{A}=9.0$ & $\begin{aligned} p_{A} & =0.382 \\
p_{B} & =0.397 \\
p & =0.778 \\
T_{A} & =0.500 \\
T_{B} & =0.500 \\
R_{A}^{e} & =0.342 \\
R_{B}^{e} & =0.340 \\
\pi^{e} & =2.335\end{aligned}$ & $\begin{aligned} p_{A} & =0.382 \\
p_{B} & =0.382 \\
p & =0.763 \\
T_{A} & =0.500 \\
T_{B} & =0.500 \\
R_{A}^{e} & =0.334 \\
R_{B}^{e} & =0.334 \\
\pi^{e} & =2.289\end{aligned}$ & $\begin{aligned} p_{A} & =0.382 \\
p_{B} & =0.368 \\
p & =0.750 \\
T_{A} & =0.500 \\
T_{B} & =0.500 \\
R_{A}^{e} & =0.327 \\
R_{B}^{e} & =0.329 \\
\pi^{e} & =2.250\end{aligned}$ \\
\hline$k_{A}=10.0$ & $\begin{aligned} p_{A} & =0.368 \\
p_{B} & =0.397 \\
p & =0.765 \\
T_{A} & =0.500 \\
T_{B} & =0.500 \\
R_{A}^{e} & =0.337 \\
R_{B}^{e} & =0.333 \\
\pi^{e} & =2.296\end{aligned}$ & $\begin{aligned} p_{A} & =0.368 \\
p_{B} & =0.382 \\
p & =0.750 \\
T_{A} & =0.500 \\
T_{B} & =0.500 \\
R_{A}^{e} & =0.329 \\
R_{B}^{e} & =0.327 \\
\pi^{e} & =2.250\end{aligned}$ & $\begin{aligned} p_{A} & =0.368 \\
p_{B} & =0.368 \\
p & =0.737 \\
T_{A} & =0.500 \\
T_{B} & =0.500 \\
R_{A}^{e} & =0.322 \\
R_{B}^{e} & =0.322 \\
\pi^{e} & =2.210\end{aligned}$ \\
\hline
\end{tabular}

Table 2. Outcomes With a Commitment to an Egalitarian Reward Structure when $V=\theta=4$. 


\begin{tabular}{|c|c|}
\hline $\begin{array}{c}\text { No Commitment to an } \\
\text { Egalitarian Reward Structure }\end{array}$ & \begin{tabular}{c} 
Commitment to an \\
Egalitarian Reward Structure \\
\hline$p_{A}=0.400$
\end{tabular} \\
$p_{B}=0.362$ & $p_{A}=0.397$ \\
$p=0.762$ & $p_{B}=0.371$ \\
$T_{A}=0.525$ & $p=0.768$ \\
$T_{B}=0.475$ & $T_{A}=0.500$ \\
$R_{A}=0.348$ & $T_{B}=0.500$ \\
$R_{B}=0.319$ & $R_{A}^{e}=0.334$ \\
$\pi$ & $=2.287$ \\
& $R_{B}^{e}=0.338$ \\
\end{tabular}

Table 3. Outcomes when $V=\theta=4, k_{A} \in[8.0,8.2]$, and $k_{B} \in[9.8,10]$. 
$100\left(\frac{\pi^{\mathrm{e}}-\pi}{\pi}\right)$

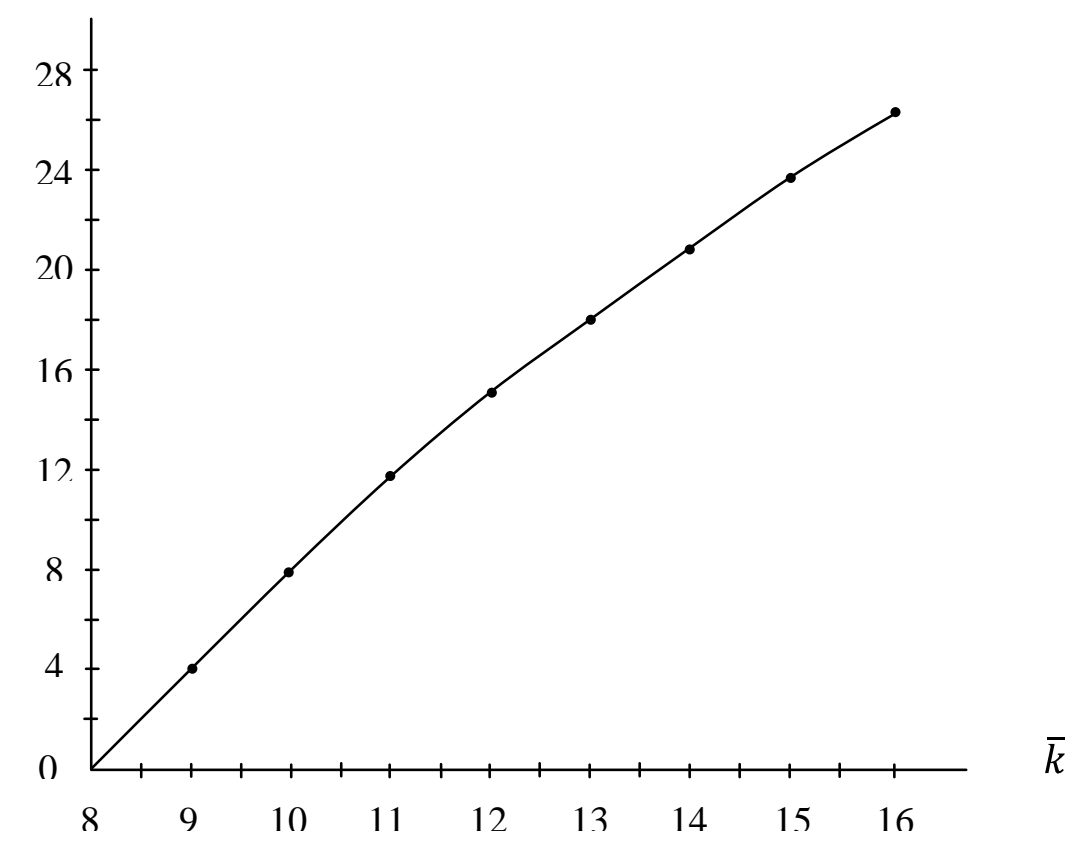

Figure 1. Principal's Gain from an Egalitarian Reward Structure as $\bar{k}$ Changes. 


$$
100\left(\frac{\pi^{\mathrm{e}}-\pi}{\pi}\right)
$$

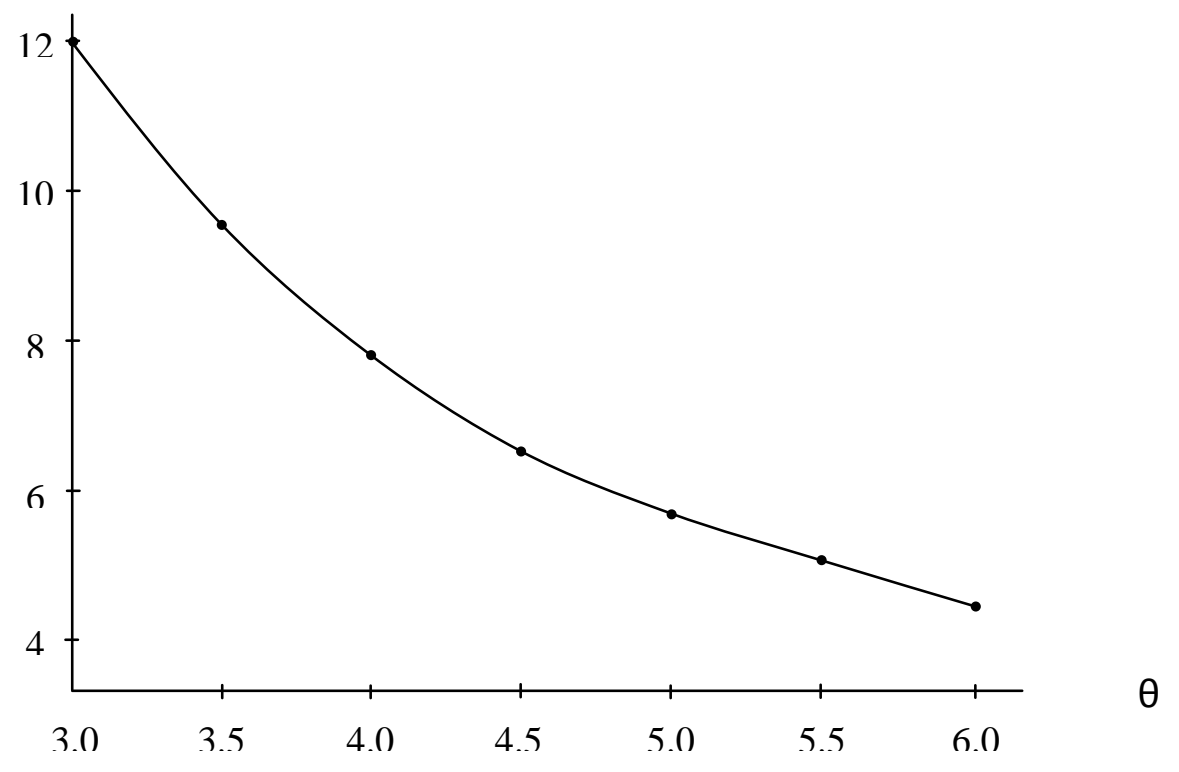

Figure 2. Principal's Gain from an Egalitarian Reward Structure as $\boldsymbol{\theta}$ Changes. 


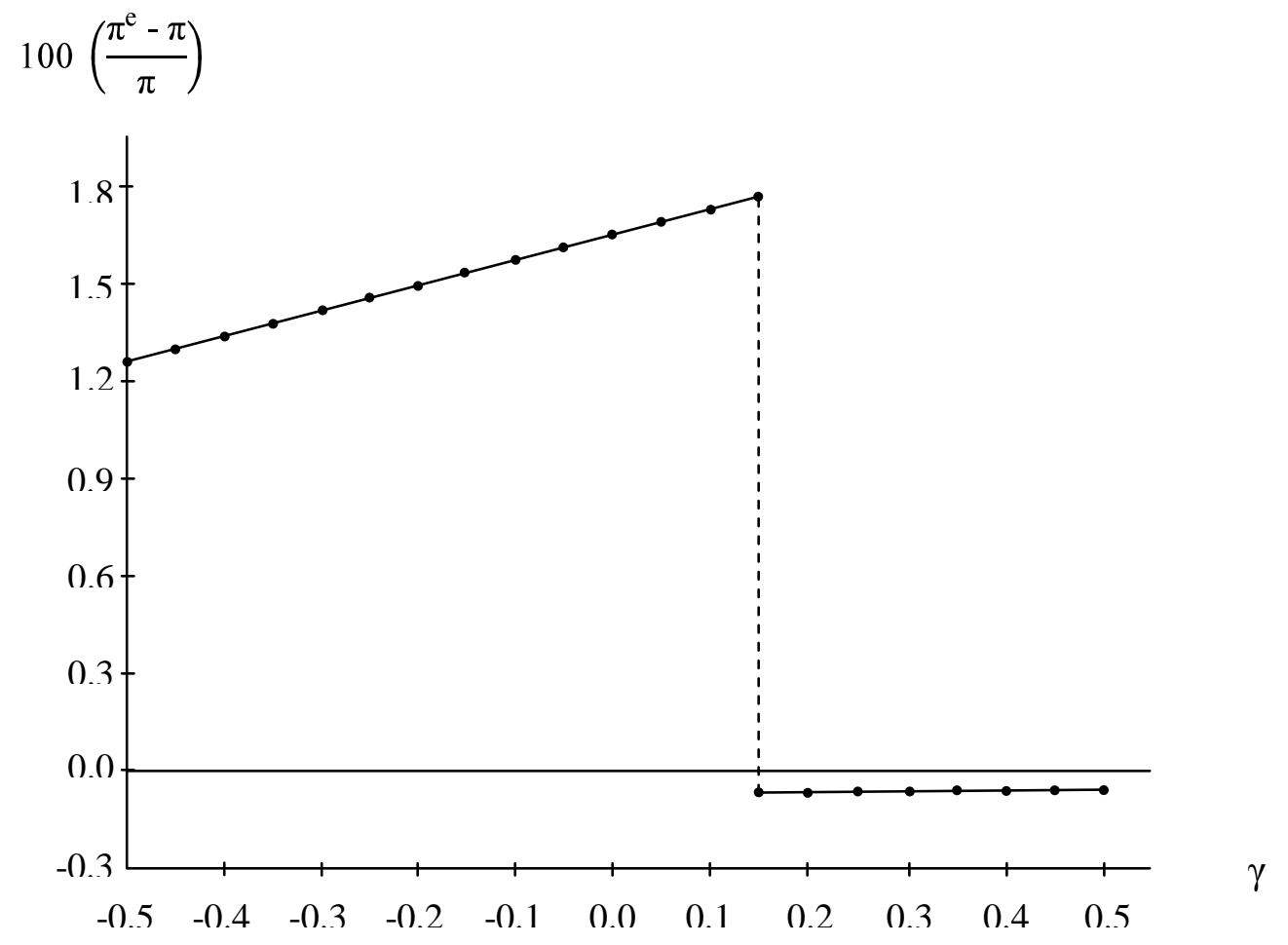

Figure 3. Principal's Gain from an Egalitarian Reward Structure as $\gamma$ Changes. 


\section{Appendix}

\section{Proof of Lemmas 1 and 2.}

When the principal hires a single agent, the agent's (expected) profit, given success probability $p$ and payment for success $T$, is $p T-\frac{k}{\theta}(p)^{\theta}$. Therefore, the agent's profit-maximizing choice of $p$, given $T$, is determined by:

$$
T=k(p)^{\theta-1}
$$

(A1) implies that the principal's expected profit is:

$$
\pi^{s}=p[V-T]=p\left[V-k(p)^{\theta-1}\right]=p V-k(p)^{\theta}
$$

Maximizing this function with respect to $p$ provides:

$$
\frac{\partial \pi^{s}}{\partial p}=V-k \theta p^{\theta-1}=0 \Rightarrow p=\left(\frac{V}{\theta k}\right)^{\frac{1}{\theta-1}}
$$

(A1), (A2), and (A3) imply that the principal's maximum profit with a single agent is:

$$
\begin{aligned}
\pi^{s} & =p\left[V-k(p)^{\theta-1}\right]=\left(\frac{V}{\theta k}\right)^{\frac{1}{\theta-1}}\left(V-\frac{V}{\theta}\right) \\
& =\left(\frac{V}{\theta k}\right)^{\frac{1}{\theta-1}} V\left(1-\frac{1}{\theta}\right)=(\theta-1)\left(\frac{V}{\theta}\right)^{\frac{\theta}{\theta-1}}\left(\frac{1}{(k)^{\frac{1}{\theta-1}}}\right) .
\end{aligned}
$$

Now suppose the principal hires two agents. Since agent $i$ 's profit given $p_{i}, p_{j}$, and $T_{i}$ is $\left(p_{i}+p_{j}\right) T_{i}-\frac{k_{i}}{\theta}\left(p_{i}\right)^{\theta}$, the profit-maximizing choices of $p_{A}$ and $p_{B}$ are determined by:

$$
T_{A}=k_{A}\left(p_{A}\right)^{\theta-1} \text { and } T_{B}=k_{B}\left(p_{B}\right)^{\theta-1}
$$

(A5) implies that the principal's problem can be stated as:

$$
\underset{\left\{p^{A}, p^{B}\right\}}{\operatorname{aximize}}\left(p_{A}+p_{B}\right)\left(V-k_{A}\left(p_{A}\right)^{\theta-1}-k_{B}\left(p_{B}\right)^{\theta-1}\right)
$$

After simplification, the necessary conditions for an interior solution to this problem can be stated as: 


$$
\begin{aligned}
& V-\theta k_{A}\left(p_{A}\right)^{\theta-1}-k_{B}\left(p_{B}\right)^{\theta-1}-k_{A} p_{B}(\theta-1)\left(p_{A}\right)^{\theta-2}=0 ; \text { and } \\
& V-\theta k_{B}\left(p_{B}\right)^{\theta-1}-k_{A}\left(p_{A}\right)^{\theta-1}-k_{B} p_{A}(\theta-1)\left(p_{B}\right)^{\theta-2}=0 .
\end{aligned}
$$

It is readily verified that these necessary conditions also are sufficient when the condition cited in Lemma 1 is satisfied.

Subtracting (A7) from (A8), and simplifying, provides:

$$
k_{A}\left(p_{A}\right)^{\theta-2}=k_{B}\left(p_{B}\right)^{\theta-2}
$$

(A5) and (A9) imply:

$$
p_{B} T_{A}=p_{A} T_{B}
$$

Rearranging (A7) provides:

$$
\begin{aligned}
& V-\theta k_{A}\left(p_{A}\right)^{\theta-1}-k_{B}\left(p_{B}\right)^{\theta-1}-\left(\frac{p_{B}(\theta-1)}{p_{A}}\right) k_{A}\left(p_{A}\right)^{\theta-1}=0 \\
\Rightarrow & V p_{A}-\theta k_{A}\left(p_{A}\right)^{\theta}-k_{B} p_{A}\left(p_{B}\right)^{\theta-1}-p_{B} \theta k_{A}\left(p_{A}\right)^{\theta-1}+k_{A} p_{B}\left(p_{A}\right)^{\theta-1}=0 \\
\Rightarrow & V p_{A}-\theta k_{A}\left(p_{A}\right)^{\theta}-p_{B} \theta k_{A}\left(p_{A}\right)^{\theta-1}=0 .
\end{aligned}
$$

(A12) follows from (A11) because, using (A9):

$$
k_{B} p_{A}\left(p_{B}\right)^{\theta-1}=k_{B}\left(p_{B}\right)^{\theta-2} p_{A} p_{B}=k_{A}\left(p_{A}\right)^{\theta-2} p_{A} p_{B}=k_{A} p_{B}\left(p_{A}\right)^{\theta-1}
$$

(A9) implies:

$$
p_{B}=\left(\frac{k_{A}}{k_{B}}\right)^{\frac{1}{\theta-2}} p_{A} .
$$

Substituting from (A13) into (A12) provides:

$$
\begin{aligned}
& V p_{A}-\theta k_{A}\left(p_{A}\right)^{\theta}-\left(\frac{k_{A}}{k_{B}}\right)^{\frac{1}{\theta-2}} \theta k_{A}\left(p_{A}\right)^{\theta}=0 \\
& \Rightarrow V-\theta k_{A}\left(p_{A}\right)^{\theta-1}-\left(\frac{k_{A}}{k_{B}}\right)^{\frac{1}{\theta-2}} \theta k_{A}\left(p_{A}\right)^{\theta-1}=0
\end{aligned}
$$




$$
\Rightarrow \quad\left(p_{A}\right)^{\theta-1}=\frac{V}{\theta k_{A}\left(1+\left(\frac{k_{A}}{k_{B}}\right)^{\frac{1}{\theta-2}}\right)} .
$$

Similarly, it is readily shown that:

$$
\left(p_{B}\right)^{\theta-1}=\frac{V}{\theta k_{B}\left(1+\left(\frac{k_{B}}{k_{A}}\right)^{\frac{1}{\theta-2}}\right)} .
$$

(A14) and (A15) imply:

$$
\begin{aligned}
& \left(p_{A}\right)^{\theta-1}=\frac{V}{\theta k_{A}}\left(\frac{\left(k_{B}\right)^{\frac{1}{\theta-2}}}{\left(k_{A}\right)^{\frac{1}{\theta-2}}+\left(k_{B}\right)^{\frac{1}{\theta-2}}}\right) ; \text { and } \\
& \left(p_{B}\right)^{\theta-1}=\frac{V}{\theta k_{B}}\left(\frac{\left(k_{A}\right)^{\frac{1}{\theta-2}}}{\left(k_{A}\right)^{\frac{1}{\theta-2}}+\left(k_{B}\right)^{\frac{1}{\theta-2}}}\right) .
\end{aligned}
$$

(A5), (A16), and (A17) imply:

$$
\begin{aligned}
& T_{A}=\frac{V}{\theta}\left(\frac{\left(k_{B}\right)^{\frac{1}{\theta-2}}}{\left(k_{A}\right)^{\frac{1}{\theta-2}}+\left(k_{B}\right)^{\frac{1}{\theta-2}}}\right) ; \text { and } \\
& T_{B}=\frac{V}{\theta}\left(\frac{\left(k_{A}\right)^{\frac{1}{\theta-2}}}{\left(k_{A}\right)^{\frac{1}{\theta-2}}+\left(k_{B}\right)^{\frac{1}{\theta-2}}}\right) .
\end{aligned}
$$

(A18) and (A19) imply:

$$
T_{A}+T_{B}=\frac{V}{\theta} .
$$

(A20) implies that the principal's profit is:

$$
\left(p_{A}+p_{B}\right)\left(V-\frac{V}{\theta}\right)=(\theta-1) \frac{V}{\theta}\left(p_{A}+p_{B}\right) .
$$

(A16) and (A17) imply:

$$
p_{A}+p_{B}=\left[\frac{V}{\theta k_{A}}\left(\frac{\left(k_{B}\right)^{\frac{1}{\theta-2}}}{\left(k_{A}\right)^{\frac{1}{\theta-2}}+\left(k_{B}\right)^{\frac{1}{\theta-2}}}\right)\right]^{\frac{1}{\theta-1}}+\left[\frac{V}{\theta k_{B}}\left(\frac{\left(k_{A}\right)^{\frac{1}{\theta-2}}}{\left(k_{A}\right)^{\frac{1}{\theta-2}}+\left(k_{B}\right)^{\frac{1}{\theta-2}}}\right)\right]^{\frac{1}{\theta-1}}
$$




$$
\begin{aligned}
& =\left(\frac{V}{\theta}\right)^{\frac{1}{\theta-1}}\left(\frac{\left(k_{B}\right)^{\frac{1}{\theta-2}}}{k_{A}}\right)^{\frac{1}{\theta-1}}\left(\frac{1}{\left(k_{A}\right)^{\frac{1}{\theta-2}}+\left(k_{B}\right)^{\frac{1}{\theta-2}}}\right)^{\frac{1}{\theta-1}} \\
& +\left(\frac{V}{\theta}\right)^{\frac{1}{\theta-1}}\left(\frac{\left(k_{A}\right)^{\frac{1}{\theta-2}}}{k_{B}}\right)^{\frac{1}{\theta-1}}\left(\frac{1}{\left(k_{A}\right)^{\frac{1}{\theta^{-2}}}+\left(k_{B}\right)^{\frac{1}{\theta^{-2}}}}\right)^{\frac{1}{\theta-1}} \\
& =\left(\frac{V}{\theta}\right)^{\frac{1}{\theta-1}}\left(\frac{1}{\left(k_{A}\right)^{\frac{1}{\theta-2}}+\left(k_{B}\right)^{\frac{1}{\theta-2}}}\right)^{\frac{1}{\theta-1}}\left[\left(\frac{\left(k_{B}\right)^{\frac{1}{\theta-2}}}{k_{A}}\right)^{\frac{1}{\theta-1}}+\left(\frac{\left(k_{A}\right)^{\frac{1}{\theta-2}}}{k_{B}}\right)^{\frac{1}{\theta-1}}\right] \\
& =\left(\frac{V}{\theta}\right)^{\frac{1}{\theta-1}}\left(\frac{1}{\left(k_{A}\right)^{\frac{1}{\theta-2}}+\left(k_{B}\right)^{\frac{1}{\theta-2}}}\right)^{\frac{1}{\theta-1}}\left[\left(\frac{\left(k_{B}\right)^{\frac{1}{(\theta-2)(\theta-1)}}}{\left(k_{A}\right)^{\frac{1}{\theta-1}}}\right)+\left(\frac{\left(k_{A}\right)^{\frac{1}{(\theta-2)(\theta-1)}}}{\left(k_{B}\right)^{\frac{1}{\theta-1}}}\right)\right] \\
& =\left(\frac{V}{\theta}\right)^{\frac{1}{\theta-1}}\left(\frac{1}{\left(k_{A}\right)^{\frac{1}{\theta-2}}+\left(k_{B}\right)^{\frac{1}{\theta-2}}}\right)^{\frac{1}{\theta-1}}\left(\frac{\left(k_{B}\right)^{\frac{1}{(\theta-2)(\theta-1)}+\frac{1}{\theta-1}}+\left(k_{A}\right)^{\frac{1}{(\theta-2)(\theta-1)}+\frac{1}{\theta-1}}}{\left(k_{A}\right)^{\frac{1}{\theta-1}}\left(k_{B}\right)^{\frac{1}{\theta-1}}}\right) \\
& =\left(\frac{V}{\theta}\right)^{\frac{1}{\theta-1}}\left(\frac{1}{\left(k_{A}\right)^{\frac{1}{\theta-2}}+\left(k_{B}\right)^{\frac{1}{\theta-2}}}\right)^{\frac{1}{\theta-1}}\left(\frac{\left(k_{B}\right)^{\frac{1}{\theta-2}}+\left(k_{A}\right)^{\frac{1}{\theta-2}}}{\left(k_{A}\right)^{\frac{1}{\theta-1}}\left(k_{B}\right)^{\frac{1}{\theta-1}}}\right) \\
& =\left(\frac{V}{\theta}\right)^{\frac{1}{\theta-1}}\left\{\left(k_{A}\right)^{\frac{1}{\theta-2}}+\left(k_{B}\right)^{\frac{1}{\theta-2}}\right\}^{\frac{\theta-2}{\theta-1}}\left[\frac{1}{\left(k_{A}\right)^{\frac{1}{\theta-1}}\left(k_{B}\right)^{\frac{1}{\theta-1}}}\right] \\
& =\left(\frac{V}{\theta}\right)^{\frac{1}{\theta-1}}\left(\left(k_{A}\right)^{\frac{1}{\theta-2}}+\left(k_{B}\right)^{\frac{1}{\theta-2}}\right)^{\frac{\theta-2}{\theta-1}}\left(k_{A} k_{B}\right)^{-\frac{1}{\theta-1}} \\
& =\left(\frac{V}{\theta}\right)^{\frac{1}{\theta-1}}\left(\left(k_{A}\right)^{\frac{1}{\theta-2}}+\left(k_{B}\right)^{\frac{1}{\theta-2}}\right)^{\frac{\theta-2}{\theta-1}}\left(k_{A} k_{B}\right)^{\left(\frac{\theta-2}{\theta-1}\right)\left(-\frac{1}{\theta-2}\right)} \\
& =\left(\frac{V}{\theta}\right)^{\frac{1}{\theta-1}}\left(\left(k_{B}\right)^{\left(-\frac{1}{\theta-2}\right)}+\left(k_{A}\right)^{\left(-\frac{1}{\theta-2}\right)}\right)^{\frac{\theta-2}{\theta-1}} \\
& =\left(\frac{V}{\theta}\right)^{\frac{1}{\theta-1}}\left(\frac{1}{\left(k_{B}\right)^{\frac{1}{\theta-2}}}+\frac{1}{\left(k_{A}\right)^{\frac{1}{\theta^{-2}}}}\right)^{\frac{\theta-2}{\theta-1}} .
\end{aligned}
$$

(A21) and (A22) imply that the principal's profit is:

$$
\pi=(\theta-1)\left(\frac{V}{\theta}\right)^{\frac{\theta}{\theta-1}}\left(\frac{1}{\left(k_{B}\right)^{\frac{1}{\theta-2}}}+\frac{1}{\left(k_{A}\right)^{\frac{1}{\theta-2}}}\right)^{\frac{\theta-2}{\theta-1}} .
$$

As the proof of Proposition 2 reveals, the equilibrium costs of agent $\mathrm{A}$ and $\mathrm{B}$ will be $\bar{k}_{A}$ 
and $\bar{k}_{B}$, respectively, in this setting. Therefore, (A23) implies:

$$
\pi=(\theta-1)\left(\frac{V}{\theta}\right)^{\frac{\theta}{\theta-1}}\left(\frac{1}{\left(\bar{k}_{B}\right)^{\frac{1}{\theta-2}}}+\frac{1}{\left(\bar{k}_{A}\right)^{\frac{1}{\theta-2}}}\right)^{\frac{\theta-2}{\theta-1}} .
$$

Without loss of generality, suppose the initial cost of agent $\mathrm{A}\left(k_{A}^{o}\right)$ is less than the corresponding initial cost of agent $\mathrm{B}$. The the principal will hire agent $\mathrm{A}$ if she decides to hire just one agent. (A4) and (A24) imply that the principal will prefer to hire two agents rather than one agent if and only if:

$$
\begin{gathered}
\left(\frac{1}{\left(\bar{k}_{B}\right)^{\frac{1}{\theta-2}}}+\frac{1}{\left(\bar{k}_{A}\right)^{\frac{1}{\theta-2}}}\right)^{\frac{\theta-2}{\theta-1}}>\frac{1}{\left(k_{A}^{o}\right)^{\frac{1}{\theta-1}}} \\
\Leftrightarrow \quad\left(\frac{1}{\left(\bar{k}_{B}\right)^{\frac{1}{\theta-2}}}+\frac{1}{\left(\bar{k}_{A}\right)^{\frac{1}{\theta-2}}}\right)^{\theta-2}>\frac{1}{k_{A}^{o}} \Leftrightarrow \frac{1}{\left(\bar{k}_{B}\right)^{\frac{1}{\theta-2}}}+\frac{1}{\left(\bar{k}_{A}\right)^{\frac{1}{\theta-2}}}>\frac{1}{\left(k_{A}^{o}\right)^{\frac{1}{\theta-2}}} .
\end{gathered}
$$

Define $\bar{k} \equiv \max \left\{\bar{k}_{A}, \bar{k}_{B}\right\}$ and define $\underline{k} \equiv \min \left\{\underline{k}_{A}, \underline{k}_{B}\right\}$. Then when $\theta>2$ :

$$
\frac{1}{\left(\bar{k}_{B}\right)^{\frac{1}{\theta-2}}}+\frac{1}{\left(\bar{k}_{A}\right)^{\frac{1}{\theta-2}}} \geq \frac{2}{(\bar{k})^{\frac{1}{\theta-2}}} \quad \text { and } \quad \frac{1}{(\underline{k})^{\frac{1}{\theta-2}}} \geq \frac{1}{\left(k_{A}^{o}\right)^{\frac{1}{\theta-2}}}
$$

(A26) implies that (A25) will hold when $\theta>2$ if:

$$
\begin{aligned}
& \frac{2}{(\bar{k})^{\frac{1}{\theta-2}}}>\frac{1}{(\underline{k})^{\frac{1}{\theta-2}}} \Leftrightarrow\left(\frac{\bar{k}}{\underline{k}}\right)^{\frac{1}{\theta-2}}<2 \\
& \Leftrightarrow \quad\left(\frac{1}{\theta-2}\right) \ln \left(\frac{\bar{k}}{\underline{k}}\right)<\ln 2 \Leftrightarrow \theta>2+\frac{\ln (\bar{k} / \underline{k})}{\ln 2} .
\end{aligned}
$$

\section{Proof of Proposition 1.}

(A16) and (A17) imply that $p_{j}$ is decreasing in $k_{j}$ for $j=A, B$ at the solution to $[\mathrm{P}]$ when

$\theta>2$. Therefore, since agent $i$ 's profit is $\left(p_{i}+p_{j}\right) T_{i}-\frac{k_{i}}{\theta}\left(p_{i}\right)^{\theta}$, agent $i$ will choose $k_{j}$ to maximize $p_{j}$ when the reward structure has already been determined. Consequently, agent $i$ will set $k_{j}=\underline{k}_{j}$ for $j \neq i, i, j \in\{A, B\}$. 


\section{Proof of Proposition 2.}

Substituting from (A5) and (A10) into (A7) provides:

$$
\begin{aligned}
& V-\theta T_{A}-T_{B}-T_{A}\left(\frac{(\theta-1) p_{B}}{p_{A}}\right)=0 \\
& \Rightarrow V p^{A}-\theta T_{A} p_{A}-T_{B} p_{A}-T_{A}(\theta-1) p_{B}=0 \\
& \Rightarrow V p_{A}=\theta T_{A}\left(p_{A}+p_{B}\right) \Rightarrow p_{A}+p_{B}=\frac{V p_{A}}{\theta T_{A}}
\end{aligned}
$$

Agent A's equilibrium profit (or rent) is:

$$
R_{A}=\left(p_{A}+p_{B}\right) T_{A}-\frac{1}{\theta} k_{A}\left(p_{A}\right)^{\theta} .
$$

Substituting from (A28) into (A29) provides:

$$
R_{A}=\frac{V p_{A}}{\theta}-\frac{1}{\theta} k_{A}\left(p_{A}\right)^{\theta}
$$

(A14) implies:

$$
\theta k_{A}\left(1+\left(\frac{k_{A}}{k_{B}}\right)^{\frac{1}{\theta-2}}\right)\left(p_{A}\right)^{\theta}=V p_{A} .
$$

Substituting from (A31) into (A30) provides:

$$
\begin{aligned}
R_{A} & =k_{A}\left(1+\left(\frac{k_{A}}{k_{B}}\right)^{\frac{1}{\theta-2}}\right)\left(p_{A}\right)^{\theta}-\frac{1}{\theta} k_{A}\left(p_{A}\right)^{\theta} \\
& =k_{A}\left(p_{A}\right)^{\theta}\left(1+\left(\frac{k_{A}}{k_{B}}\right)^{\frac{1}{\theta-2}}-\frac{1}{\theta}\right) .
\end{aligned}
$$

From (A14):

$$
k_{A}\left(p_{A}\right)^{\theta}=\left(\frac{V}{\theta\left(1+\left(\frac{k_{A}}{k_{B}}\right)^{\frac{1}{\theta-2}}\right)}\right)^{\frac{\theta}{\theta-1}} .
$$

Substituting from (A33) into (A32) provides: 


$$
\begin{aligned}
R_{A}= & \left(\frac{V}{\theta\left(1+\left(\frac{k_{A}}{k_{B}}\right)^{\frac{1}{\theta-2}}\right)}\right)^{\frac{\theta}{\theta-1}}\left(1+\left(\frac{k_{A}}{k_{B}}\right)^{\frac{1}{\theta-2}}-\frac{1}{\theta}\right) \\
& =\left(\frac{V}{\theta}\right)^{\frac{\theta}{\theta-1}}\left(\frac{1}{1+\left(\frac{k_{A}}{k_{B}}\right)^{\frac{1}{\theta-2}}}\right)^{\frac{\theta}{\theta-1}}\left(1+\left(\frac{k_{A}}{k_{B}}\right)^{\frac{1}{\theta-2}}-\frac{1}{\theta}\right) .
\end{aligned}
$$

Let $\frac{1}{x}=\left(\frac{k_{A}}{k_{B}}\right)^{\frac{1}{\theta-2}}$. Then (A34) implies:

$$
R_{A}=\left(\frac{V}{\theta}\right)^{\frac{\theta}{\theta-1}}\left(\frac{1}{1+\frac{1}{x}}\right)^{\frac{\theta}{\theta-1}}\left(1+\frac{1}{x}-\frac{1}{\theta}\right)
$$

(A35) implies:

$$
\begin{aligned}
\ln R_{A}= & \left(\frac{\theta}{\theta-1}\right) \ln \left(\frac{V}{\theta}\right)+\left(\frac{\theta}{\theta-1}\right)\left[\ln (1)-\ln \left(1+\frac{1}{x}\right)\right]+\ln \left(1+\frac{1}{x}-\frac{1}{\theta}\right) \\
= & \left(\frac{\theta}{\theta-1}\right) \ln \left(\frac{V}{\theta}\right)-\left(\frac{\theta}{\theta-1}\right) \ln \left(\frac{x+1}{x}\right)+\ln \left(\frac{x \theta+\theta-x}{x \theta}\right) \\
= & \left(\frac{\theta}{\theta-1}\right) \ln \left(\frac{V}{\theta}\right)-\left(\frac{\theta}{\theta-1}\right)[\ln (x+1)-\ln (x)] \\
& \quad+\ln (x \theta+\theta-x)-\ln (x \theta) .
\end{aligned}
$$

(A36) implies:

$$
\begin{aligned}
\frac{d \ln R_{A}}{d x} & =-\left(\frac{\theta}{\theta-1}\right)\left(\frac{1}{x+1}-\frac{1}{x}\right)+\frac{\theta-1}{x \theta+\theta-x}-\frac{1}{x} \\
& =\left(\frac{\theta}{\theta-1}\right) \frac{1}{x(x+1)}-\frac{\theta}{x(x \theta+\theta-x)} \\
& =\frac{\theta}{\theta(\theta-1)(1+x)(x \theta+\theta-x)}>0 .
\end{aligned}
$$

(A37) implies $\frac{d R_{A}}{d x}>0$. Also, $\frac{d x}{d k_{B}}>0$ when $\theta>2$ because $x=\left(\frac{k_{B}}{k_{A}}\right)^{\frac{1}{\theta-2}}$. Therefore: 


$$
\frac{d R_{A}}{d k_{B}}=\left(\frac{d R_{A}}{d x}\right)\left(\frac{d x}{d k_{B}}\right)>0 \text { for all } k_{A} \in\left[\underline{k}_{A}, \bar{k}_{A}\right] \text { and } k_{B} \in\left[\underline{k}_{B}, \bar{k}_{B}\right] \text {. }
$$

(A38) implies that each agent will undertake the maximum feasible level of sabotage, i.e., $k_{A}=\bar{k}_{A}$ and $k_{B}=\bar{k}_{B}$.

\section{Proof of Proposition 3.}

Let $T_{A}=T_{B}=T$. Then (A5) implies:

$$
T=k_{A}\left(p_{A}\right)^{\theta-1}=k_{B}\left(p^{B}\right)^{\theta-1} \Rightarrow p_{B}=\left(\frac{k_{A}}{k_{B}}\right)^{\frac{1}{\theta-1}} p_{A} .
$$

Recall that the principal maximizes:

$$
\pi^{e}=\left[p_{A}(T)+p_{B}(T)\right]\left(V_{S}-T-T\right) .
$$

Substituting from (A39) into (A40) provides:

$$
\pi^{e}=p_{A}\left(1+\left(\frac{k_{A}}{k_{B}}\right)^{\frac{1}{\theta-1}}\right)\left(V-2 k_{A}\left(p_{A}\right)^{\theta-1}\right) .
$$

(A41) implies:

$$
\frac{\partial \pi^{e}}{\partial p_{A}}=\left(1+\left(\frac{k_{A}}{k_{B}}\right)^{\frac{1}{\theta-1}}\right)\left(V-2 \theta k_{A}\left(p_{A}\right)^{\theta-1}\right) .
$$

(A42) implies:

$$
\frac{\partial \pi^{e}}{\partial p_{A}}=0 \quad \Leftrightarrow \quad V-2 \theta k_{A}\left(p_{A}\right)^{\theta-1}=0 \quad \Leftrightarrow \quad p_{A}=\left(\frac{V}{2 \theta k_{A}}\right)^{\frac{1}{\theta-1}} .
$$

Note that $\frac{\partial^{2} \pi^{e}}{\partial\left(p_{A}\right)^{2}}<0$ since $\theta>2$.

Similarly:

$$
\frac{\partial \pi^{e}}{\partial p_{B}}=0 \quad \Leftrightarrow \quad p_{B}=\left(\frac{V}{2 \theta k_{B}}\right)^{\frac{1}{\theta-1}}
$$

(A39) and (A43) imply:

$$
T=k_{A}\left(p_{A}\right)^{\theta-1}=k_{A}\left(\frac{V}{2 \theta k_{A}}\right)=\frac{V}{2 \theta} .
$$


(A43) and (A44) imply:

$$
\frac{\partial p_{A}}{\partial k_{B}}=0 \text { and } \frac{\partial p_{B}}{\partial k_{B}}<0 .
$$

From (A45), the rate at which agent A's profit increases with $k_{B}$ is:

$$
\begin{aligned}
\frac{\partial R_{A}}{\partial k_{B}} & =\frac{\partial}{\partial k_{B}}\left(\left(p_{A}+p_{B}\right) \frac{V}{2 \theta}-\frac{1}{\theta} k_{A}\left(p_{A}\right)^{\theta}\right) \\
& =\left(\frac{\partial p_{A}}{\partial k_{B}}+\frac{\partial p_{B}}{\partial k_{B}}\right) T-k_{A}\left(p_{A}\right)^{\theta-1} \frac{\partial p_{A}}{\partial k_{B}} .
\end{aligned}
$$

(A46) and (A47) imply:

$$
\frac{\partial R_{A}}{\partial k_{B}}=\left(\frac{\partial p_{B}}{\partial k_{B}}\right) T<0
$$

(A48) implies that if the principal commits to implement an egalitarian reward structure, the agents will choose $k_{A}=\underline{k}_{A}$ and $k_{B}=\underline{k}_{B}$.

\section{Proof of Proposition 4.}

Let $\pi^{e}\left(k_{A}, k_{B}\right)$ denote the principal's (maximized) profit when she commits ex ante to an egalitarian reward structure, and let $\pi\left(k_{A}, k_{B}\right)$ denote the corresponding profit in the absence of this commitment, given $\left(k_{A}, k_{B}\right)$. Proposition 3 implies that $\left(k_{A}, k_{B}\right)=\left(\underline{k}_{A}, \underline{k}_{B}\right)$ in equilibrium under an egalitarian reward structure. Proposition 2 implies that $\left(k_{A}, k_{B}\right)=\left(\bar{k}_{A}, \bar{k}_{B}\right)$ in equilibrium in the absence of a commitment to an egalitarian reward structure. We seek to show that:

$$
\pi\left(\bar{k}_{A}, \bar{k}_{B}\right)<\pi^{e}\left(\underline{k}_{A}, \underline{k}_{B}\right) .
$$

From (A6) and the envelope theorem:

$$
\frac{\partial \pi\left(k_{A}, k_{B}\right)}{\partial k_{i}}=-\left(p_{A}+p_{B}\right)\left(p_{i}\right)^{\theta-1}<0 \text { for } i=A, B
$$

(A43) - (A45) imply that under an egalitarian reward structure:

$$
p_{A}=\left(\frac{V}{2 \theta k_{A}}\right)^{\frac{1}{\theta-1}} ; \quad p_{B}=\left(\frac{V}{2 \theta k_{B}}\right)^{\frac{1}{\theta-1}} ; \quad \text { and } \quad T=\frac{V}{2 \theta} .
$$

(A50) implies: 


$$
\pi^{e}\left(k_{A}, k_{B}\right)=\left(p_{A}+p_{B}\right)(V-2 T)=\left(\left(\frac{V}{2 \theta k_{A}}\right)^{\frac{1}{\theta-1}}+\left(\frac{V}{2 \theta k_{B}}\right)^{\frac{1}{\theta-1}}\right) V\left(1-\frac{1}{\theta}\right) .
$$

It is apparent from (A51) that when $\theta>2$ :

$$
\frac{d \pi^{e}\left(k_{A}, k_{B}\right)}{d k_{i}}<0 \text { for } i=A, B
$$

Consider $(\widehat{k}, \widehat{k}) \in\left(\underline{k}_{A}, \bar{k}_{A}\right) \cap\left(\underline{k}_{B}, \bar{k}_{B}\right)$. Then, (A49) implies:

$$
\pi\left(\bar{k}_{A}, \bar{k}_{B}\right)<\pi\left(\widehat{k}, \bar{k}_{B}\right)<\pi(\widehat{k}, \widehat{k})
$$

(A52) implies:

$$
\pi^{e}(\widehat{k}, \widehat{k})<\pi^{e}\left(\underline{k}_{A}, \widehat{k}\right)<\pi^{e}\left(\underline{k}_{A}, \underline{k}_{B}\right)
$$

(A10) and (A13) imply that $T_{A}=T_{B}$ when $k_{A}=k_{B}$. Therefore, $\pi(\widehat{k}, \widehat{k})=\pi^{e}(\widehat{k}, \widehat{k})$. Consequently, (A53) and (A54) imply:

$$
\pi\left(\bar{k}_{A}, \bar{k}_{B}\right)<\pi(\widehat{k}, \widehat{k})=\pi^{e}(\widehat{k}, \widehat{k})<\pi^{e}\left(\underline{k}_{A}, \underline{k}_{B}\right)
$$

\section{Proof of Proposition 5.}

Proposition 3 implies that $\left(k_{A}, k_{B}\right)=\left(\underline{k}_{A}, \underline{k}_{B}\right)$ in equilibrium when the principal commits ex ante to implement an egalitarian reward structure. Proposition 2 implies that $\left(k_{A}, k_{B}\right)=$ $\left(\bar{k}_{A}, \bar{k}_{B}\right)$ in equilibrium in the absence of a such a commitment. Since $\bar{k}_{A}=\bar{k}_{B}$, the same equilibrium costs arise absent a commitment to an egalitarian reward structure that arise in the presence of such a commitment when the equilibrium costs for agent $\mathrm{A}$ and agent $\mathrm{B}$ are $k_{A}=\bar{k}_{A}$ and $k_{B}=\bar{k}_{B}$, respectively. Therefore, since $\pi^{e}\left(\underline{k}_{A}, \underline{k}_{B}\right)>\pi^{e}\left(\bar{k}_{A}, \bar{k}_{B}\right)$ from Proposition 4, it suffices to show that:

$$
R_{i}^{e}\left(\underline{k}_{A}, \underline{k}_{B}\right)>R_{i}^{e}\left(\bar{k}_{A}, \bar{k}_{B}\right) \text { for } i=A, B
$$

where $\quad R_{i}^{e}\left(k_{A}, k_{B}\right)=\left(p_{A}+p_{B}\right) T-\frac{k_{i}}{\theta}\left(p_{i}\right)^{\theta} \quad$ for $i=A, B$.

(A45) and (A57) imply: 


$$
R_{A}^{e}\left(k_{A}, k_{B}\right)=\left(p_{A}+p_{B}\right) \frac{V}{2 \theta}-\frac{1}{\theta}\left(p_{A}\right) \frac{V}{2 \theta}=\frac{V}{2 \theta}\left(p_{A}\left(1-\frac{1}{\theta}\right)+p_{B}\right) .
$$

(A50) and (A58) imply that $R_{A}^{e}\left(k_{A}, k_{B}\right)$ is decreasing in both $k_{A}$ and $k_{B}$ when $\theta>2$. Therefore, $R_{A}^{e}\left(\underline{k}_{A}, \underline{k}_{B}\right)>R_{A}^{e}\left(\bar{k}_{A}, \bar{k}_{B}\right)$. Analogous arguments reveal that $R_{B}^{e}\left(\underline{k}_{A}, \underline{k}_{B}\right)>R_{B}^{e}\left(\bar{k}_{A}, \bar{k}_{B}\right)$. 


\section{References}

Agell, Jonas, and Per Lundborg, "Theories of Pay and Unemployment: Survey Evidence from Swedish Manufacturing Firms," Scandinavian Journal of Economics, 97(2), June 1995, 295-307.

Auriol, Emmanuelle, Guido Friebel, and Lambros Pechlivanos, "Career Concerns in Teams," Journal of Labor Economics, 20(2), April 2002, 289-307.

Baker, Michael and Nicole Fortin, "Comparable Worth in a Decentralized Labour Market: The Case of Ontario," Canadian Journal of Economics, 37(4), November 2004, 850-878.

Bartling, Bjorn and Ferdinand von Siemens, "Equal Sharing Rules in Partnerships," University of Zurich mimeo, September 2007 (http://epub.ub.uni-muenchen.de/2027/1/TeamIncentivesMunich.pdf).

Bevia, Carmen and Luis Corchon, "Rational Sabotage in Cooperative Production with Heterogeneous Agents," Berkeley Electronic Press Topics in Theoretical Economics, 6(1), 2006, Article 16.

Bewley, Truman, Why Wages Don't Fall During a Recession. Cambridge, MA: Harvard University Press, 1999.

Blinder, Alan, and Don Choi, "A Shred of Evidence on Theories of Wage Stickiness," Quarterly Journal of Economics, 195(4), November 1990, 1003-1015.

Borland, Jeff, "Equal Pay Thirty Years On," Australian Economic Review, 32(3), September 1999, 265-272.

Campbell, Carl and Kunal Kamlani, "The Reasons for Wage Rigidity: Evidence from A Survey of Firms," Quarterly Journal of Economics, 112(3) August 1997, 759-789.

Chen, Kong-Pin, "Sabotage in Promotion Tournaments," Journal of Law, Economics and Organization, 19(1), April 2003, 119-140.

Dye, Ronald, "The Trouble with Tournaments," Economic Inquiry, 22(1), January 1984, 147-149.

Encinosa, William, Martin Gaynor, and James Rebitzer, "The Sociology of Groups and the Economics of Incentives: Theory and Evidence on Compensation Systems," Journal of Economic Behavior and Organization, 62(2), February 2007, 187-214.

Farrell, Joseph and Suzanne Scotchmer, "Partnerships," Quarterly Journal of Economics, 103(2), May 1988, 279-297. 
Heide, Ingeborg, "Supranational Action Against Sex Discrimination: Equal Pay and Equal Treatment in the European Union," International Labour Review, 138(4), December 1999, $381-410$.

Itoh, Hideshi, "Incentives to Help in Multi-Agent Situations," Econometrica, 59(3), May 1991, 611-636.

Lazear, Edward, "Pay Equality and Industrial Politics," Journal of Political Economy, 97(3), June 1989, 561-580.

Maatta, Paula, Equal Pay Policies: International Review of Selected Developing and Developed Countries. Geneva: International Labour Organization, 2000. (Available at http://www. ilo.org/public/english/dialogue/ifpdial/publ/infocus/equalpay/index.htm.)

Mui, Vai-Lam, "The Economics of Envy," Journal of Economic Behavior and Organization, 26(3), May 1995, 311-336.

Münster, Johannes, "Selection Tournaments, Sabotage, and Participation," Journal of Economics and Management Strategy, 16(4), Winter 2007, 943-970.

Sappington, David, "Limited Liability Contracts Between Principal and Agent," Journal of Economic Theory, 29(1), February 1983, 1-21. 